This item was submitted to Loughborough's Research Repository by the author.

Items in Figshare are protected by copyright, with all rights reserved, unless otherwise indicated.

\title{
Semiclassical quantization of the hydrogen atom in crossed electric and magnetic fields
}

PLEASE CITE THE PUBLISHED VERSION

http://doi.org/10.1103/PhysRevA.67.063411

\section{PUBLISHER}

(C) The American Physical Society

\section{VERSION}

VoR (Version of Record)

\section{PUBLISHER STATEMENT}

This work is made available according to the conditions of the Creative Commons Attribution-NonCommercialNoDerivatives 4.0 International (CC BY-NC-ND 4.0) licence. Full details of this licence are available at: https://creativecommons.org/licenses/by-nc-nd/4.0/

\section{LICENCE}

CC BY-NC-ND 4.0

\section{REPOSITORY RECORD}

Bartsch, Thomas, Jorg Main, and Gunter Wunner. 2019. "Semiclassical Quantization of the Hydrogen Atom in Crossed Electric and Magnetic Fields”. figshare. https://hdl.handle.net/2134/37808. 


\title{
Semiclassical quantization of the hydrogen atom in crossed electric and magnetic fields
}

\author{
Thomas Bartsch, Jörg Main, and Günter Wunner \\ Institut für Theoretische Physik 1, Universität Stuttgart, D-70550 Stuttgart, Germany \\ (Received 6 December 2002; revised manuscript received 21 February 2003; published 27 June 2003)
}

\begin{abstract}
The $S$-matrix theory formulation of closed-orbit theory recently proposed by Granger and Greene is extended to atoms in crossed electric and magnetic fields. We present a semiclassical quantization of the hydrogen atom in crossed fields, which succeeds in resolving individual lines in the spectrum, but is restricted to the strongest lines of each $n$ manifold. By means of a detailed semiclassical analysis of the quantum spectrum, we demonstrate that it is the abundance of bifurcations of closed orbits that precludes the resolution of finer details. They necessitate the inclusion of uniform semiclassical approximations into the quantization process. Uniform approximations for the generic types of closed-orbit bifurcations are derived, and a general method for including them in a high-resolution semiclassical quantization is devised.
\end{abstract}

DOI: 10.1103/PhysRevA.67.063411

PACS number(s): 32.60.+i, 03.65.Sq, 32.80.-t, 05.45.-a

\section{INTRODUCTION}

Closed-orbit theory was first introduced by Du and Delos [1] and Bogomolny [2] some 20 years ago to interpret the modulations observed in the photoabsorption spectra of hydrogenic Rydberg atoms in a magnetic field close to the ionization threshold. Since that time, it turned out to be a powerful and flexible tool for the semiclassical interpretation of a variety of spectra. It has been used to describe atoms in electric [3] as well as parallel $[4,5]$ or crossed [6-8] electric and magnetic fields. In the case of nonhydrogenic atoms, the influence of the ionic core can be modeled either by means of an effective classical potential $[9,10]$ or in terms of quantum defects $[11,12]$. Recently, closed-orbit theory has even been shown to be applicable to the spectra of simple molecules in external fields [13].

A complete description of photoabsorption spectra requires the calculation of the energies $E_{n}$ of the excited atomic states and the strengths of the spectral lines, which is characterized by the dipole matrix elements $\langle i|D| n\rangle$ between the initial state $|i\rangle$ and the Rydberg state $|n\rangle$, where $D$ is the component of the dipole operator describing the polarization of the exciting laser field. These quantities are neatly summarized in the response function

$$
g(E)=-\frac{1}{\pi}\langle i|D G(E) D| i\rangle=-\frac{1}{\pi} \sum_{n} \frac{\left.\langle i|D| n\rangle\right|^{2}}{E-E_{n}+i \epsilon},
$$

where

$$
G(E)=\sum_{n} \frac{|n\rangle\langle n|}{E-E_{n}+i \epsilon}
$$

denotes retarded Green's function.

The closed-orbit theory provides a semiclassical approximation to the quantum response function (1), which splits into a smooth part and an oscillatory part of the form

$$
g^{\mathrm{osc}}(E)=\sum_{\text {c.o. }} \mathcal{A}_{\text {c.o. }}(E) e^{i S_{\text {c.o. }}(E)}
$$

where the sum extends over all classical closed orbits starting from the nucleus and returning to it after having been deflected by the external fields, $S_{\text {c.o. }}$ is the classical action of the closed orbit, and the amplitude $\mathcal{A}_{\text {c.o. }}$ describes its stability and its starting and returning directions. Its precise form depends on the geometry of the external fields. In Sec. III, it will be specified for crossed-field systems without a rotational symmetry.

Although the closed-orbit sum (3) appears to provide a straightforward means of calculating the response function from the classical closed orbits, this is actually not the case because the sum usually diverges for real energies $E$. Thus, the quantal information cannot be extracted directly from the semiclassical expansion. One particular and widely applicable method to overcome the convergence problems of the closed-orbit sum is semiclassical quantization by harmonic inversion $[14,15]$. For the hydrogen atom in a magnetic field, this method has been shown [16] to be capable of extracting semiclassical eigenenergies and transition matrix elements from a closed-orbit sum.

In the present paper we will investigate how these results can be generalized to the hydrogen atom in crossed electric and magnetic fields. This problem is considerably harder than the treatment of the diamagnetic hydrogen atom, which possesses a rotational symmetry around the field axis. Due to that symmetry, in classical mechanics the angular momentum around the field axis is conserved. So is, in quantum mechanics, the magnetic quantum number $m$. In crossed fields, the rotational symmetry is broken. As a consequence, the selection rules for the $m$ quantum number no longer hold, and a multitude of additional lines appears in the quantum spectrum. At the same time, the determination of classical closed orbits gets significantly more difficult because three nonseparable degrees of freedom have to be dealt with. A detailed description of the intricate pattern of closed orbits and their bifurcations was given in an accompanying paper [17]. That data forms the basis of the present work, where the semiclassical treatment of the crossed-fields hydrogen atom will be dealt with, and we will freely use the nomenclature introduced in Ref. [17].

After the essential properties of the crossed-fields Hamiltonian have been summarized in Sec. II, we start, in Sec. III, 
with a derivation of the closed-orbit formula (3) in the context of the $S$-matrix formulation of closed-orbit theory introduced recently by Granger and Greene [18]. We show that the framework can be extended to the crossed-fields situation, and we clarify some misleading conclusions arrived at in Ref. [18]. Section IV describes the quantum spectrum under study, and Sec. V compares it to a semiclassical spectrum in low resolution. In Sec. VI, the results of a high-resolution semiclassical quantization using the technique of harmonic inversion are presented. The semiclassical spectrum correctly identifies the strongest spectral lines, but it fails to describe finer details of the quantum spectrum. In Sec. VII, we compare a quantum recurrence spectrum to the classical data to show that the principal source of this difficulty lies in the abundance of closed-orbit bifurcations. Uniform approximations provide a tool to cope with the divergences introduced into semiclassical spectra by bifurcations of classical orbits. A general technique for their construction is described in Sec. VIII, and uniform approximations for the two types of generic codimension-1 bifurcations identified in Ref. [17] are derived. Finally, in Sec. IX we demonstrate how uniform approximations can be incorporated into recurrence spectra, thus paving the way for their inclusion into the highresolution semiclassical quantization by harmonic inversion.

\section{THE CLASSICAL HAMILTONIAN}

Throughout this work, we will assume the magnetic field to be directed along the $z$ axis and the electric field to be directed along the $x$ axis. In atomic units, the Hamiltonian describing the motion of the atomic electron then reads

$$
H=\frac{1}{2} \boldsymbol{p}^{2}-\frac{1}{r}+\frac{1}{2} B L_{z}+\frac{1}{8} B^{2} \rho^{2}+F x,
$$

where $B$ and $F$ denote the magnetic- and electric-field strengths, respectively, $r^{2}=x^{2}+y^{2}+z^{2}, \rho^{2}=x^{2}+y^{2}$, and $L_{z}$ is the $z$ component of the angular momentum vector. By virtue of the scaling properties of Hamiltonian (4), if all classical quantities are multiplied by suitable powers of the scaling parameter

$$
w \equiv B^{-1 / 3},
$$

the dynamics can be shown not to depend on the energy $E$ and the field strengths $B$ and $F$ separately, but only on the scaled energy $\widetilde{E}=w^{2} E$ and the scaled electric-field strength $\widetilde{F}=w^{4} F$. In particular, classical actions scale according to $S=w \widetilde{S}$. Thus, the semiclassical limit of large classical actions corresponds to the limit of large $w$.

The way of recording a quantum spectrum that is best suited for semiclassical investigations is scaled-energy spectroscopy. A spectrum then consists of a list of the scaling parameters $w_{n}$ characterizing the quantum states for given scaled energy $\widetilde{E}$ and scaled electric-field strength $\widetilde{F}$. Scaledenergy spectroscopy offers the advantage that the underlying classical dynamics does not change across the spectrum. It will be adopted throughout this work.

\section{THE $S$-MATRIX FORMULATION OF CLOSED-ORBIT THEORY}

\section{A. General formalism}

The basic observation fundamental to all of the closedorbit theory is a partition of space into physically distinct regions. In the core region close to the nucleus, the Rydberg electron interacts in a complicated manner with all electrons of the ionic core. This interaction is manifestly quantum mechanical in nature, it cannot be described in the framework of semiclassical theories. On the other hand, the interaction of the Rydberg electron with the external fields is much weaker in the core region than its interaction with the core, so that the fields can safely be neglected. Therefore, a description of the core obtained in the field-free case can be used. In particular, the initial state of the photoabsorption process is assumed to be localized in the core region and not to be influenced by the external fields.

In the long-range region far away from the nucleus, on the other hand, the external fields play a dominant role, whereas there is no interaction with the ionic core except for the Coulomb attraction of its residual charge. In this region, the dynamics of the Rydberg electron is well suited for a semiclassical description. It is independent of the details of the ionic core.

In order to establish a link between the dynamics in the core and long-range regions, a matching region is assumed to exist at intermediate distances from the nucleus where both the external fields and the interaction with the core are negligible. Thus, in the matching region the simple physics of an electron subject to the residual Coulomb field of the core is observed.

Recently, Granger and Greene [18] proposed a formulation of the theory based on ideas borrowed from quantumdefect theory. Their formulation achieves a clear separation between properties of the external field configuration and the ionic core, which are encoded in separate $S$ matrices. Suitable approximations to the core and the long-range $S$ matrices can be derived independently. Therefore, the formalism can be expected to allow a generalization of closed-orbit theory to atoms with ionic cores exhibiting more complicated internal dynamics than have been treated so far.

The derivation given by Granger and Greene treated the case of an atom in a magnetic field only. It will now be extended in such a way that it holds for combined electric and magnetic fields with arbitrary field configurations. To this end, the ansatz and basic formulas of Granger and Greene's theory will be summarized in this section. A more detailed treatment can be found in their paper [18]. In subsequent sections, we will then turn to a discussion of the long-range scattering matrices pertinent to crossed external fields.

To lay the foundation for a definition of the $S$ matrices, we pick a basis set $\Psi_{k}^{\text {core }}$ and $\Psi_{k}^{\mathrm{LR}}$ of wave functions of the Rydberg electron valid in the core and long-range regions, respectively, and expand in terms of spherical harmonics:

$$
\Psi_{k}^{\mathrm{core}(\mathrm{LR})}(r, \vartheta, \varphi)=\frac{1}{r} \sum_{k^{\prime}} Y_{k^{\prime}}(\vartheta, \varphi) F_{k^{\prime} k}^{\mathrm{core}(\mathrm{LR})}(r)
$$


The channel index $k$ is to be understood as a double index $(l, m)$ characterizing the spherical harmonics. When studying a complicated atom with more than one relevant state of the core, additional information can be included in the channel functions $Y_{k}$.

In the matching region, the radial function matrices $F^{\text {core }}$ and $F^{\mathrm{LR}}$ can both be expressed in terms of radial Coulomb functions. We use the functions $f_{k}^{+}(r)$ and $f_{k}^{-}(r)$ satisfying outgoing- and incoming-wave boundary conditions, respectively, given by Ref. [19] and choose the radial functions to be of the form [47]

$$
\begin{aligned}
& F_{k^{\prime} k}^{\mathrm{core}}(r)=-i\left[f_{k^{\prime}}^{+}(r) S_{k^{\prime} k}^{\text {core }}-f_{k^{\prime}}^{-}(r) \delta_{k^{\prime} k}\right], \\
& F_{k^{\prime} k}^{\mathrm{LR}}(r)=-i\left[f_{k^{\prime}}^{+}(r) \delta_{k^{\prime} k}-f_{k^{\prime}}^{-}(r) S_{k^{\prime} k}^{\mathrm{LR}}\right] .
\end{aligned}
$$

Physically, these choices mean that the basis function $\Psi_{k}^{\text {core }}$ is a superposition of a single incoming wave in channel $k$ and the outgoing waves in different channels produced from it by scattering off the core. Similarly, $\Psi_{k}^{\mathrm{LR}}$ consists of an outgoing wave in channel $k$ and the returning waves generated by scattering off the external fields. The scattering matrices $S^{\text {core }}$ and $\underline{S}^{\mathrm{LR}}$ thus summarize the physical properties of the core and the external fields, respectively. They are determined by the condition that the radial functions obey suitable boundary conditions, i.e., $F^{\text {core }}$ is regular at the origin, whereas $F^{\mathrm{LR}}$ vanishes or satisfies outgoing-wave boundary conditions at infinity for bound and free states, respectively. For hydrogen, $\underline{S}^{\text {core }}$ is the identity matrix.

Following previous work by Robicheaux [20], Granger and Greene derive the following expression for the response function (1):

$$
\begin{aligned}
g= & i \underline{d}^{\dagger}\left[\underline{1}+2\left(\underline{S}^{\mathrm{core}} \underline{S}^{\mathrm{LR}}\right)+2\left(\underline{S}^{\mathrm{core}} \underline{S}^{\mathrm{LR}}\right)^{2}+2\left(\underline{S}^{\mathrm{core}} \underline{S}^{\mathrm{LR}}\right)^{3}\right. \\
& +\cdots] \underline{d},
\end{aligned}
$$

where the vector $\underline{d}$ comprises the energy-dependent dipole matrix elements

$$
d_{k}(E)=\left\langle\Psi_{k}^{\text {core }}(E)|D| i\right\rangle
$$

between the initial state and the core-region channel wave functions. For hydrogen they can be computed explicitly (see, e.g., Ref. [1] or [21]).

The terms of series (9) embody contributions from paths where the Rydberg electron takes $0,1,2$, etc. trips out into the long-range region and back to the core before interfering with the initial outgoing wave. In the semiclassical approximation, $\underline{S}^{\mathrm{LR}}$ will be given in terms of closed orbits. A returning wave is associated with each returning classical orbit. By a general ionic core, it is scattered into all directions. The parts of the wave scattered into the outgoing direction of a closed orbit will then follow this orbit until they return to the core again. Thus, core scattering leads to a concatenation of different closed orbits $[11,12]$. In hydrogen, the Coulomb center scatters the incoming wave back into its direction of incidence, so that there is no coupling of closed orbits. Terms describing repeated scattering off the external fields are therefore absent from the sum, and the hydrogen response function can be decomposed into a smooth part

$$
g_{0}=i \underline{d^{\dagger}} \underline{d},
$$

which is the same as in the field-free case and contains "direct" contributions where the electron does not scatter off the external fields at all, and an oscillatory part

$$
g^{\mathrm{osc}}=2 i \underline{d}^{\dagger} \underline{S}^{\mathrm{LR}} \underline{d}
$$

generated by the electron going out into the long-range region and being scattered back to the nucleus. It is this part that describes the impact of the external fields.

The basis for a semiclassical approximation is provided by the retarded Green's function $G\left(\boldsymbol{x}, \boldsymbol{x}^{\prime} ; E\right)$ describing the propagation of the electron from $\boldsymbol{x}^{\prime}$ to $\boldsymbol{x}$ at the energy $E$. It can be expanded in terms of the channel functions as

$$
G\left(\boldsymbol{x}, \boldsymbol{x}^{\prime} ; E\right)=\frac{1}{r r^{\prime}} \sum_{k k^{\prime}} Y_{k}(\vartheta, \varphi) \widetilde{G}_{k k^{\prime}}\left(r, r^{\prime} ; E\right) Y_{k^{\prime}}^{*}\left(\vartheta^{\prime}, \varphi^{\prime}\right)
$$

with

$$
\widetilde{G}_{k k^{\prime}}\left(r, r^{\prime} ; E\right)=r r^{\prime}\left\langle k\left|G\left(\boldsymbol{x}, \boldsymbol{x}^{\prime} ; E\right)\right| k^{\prime}\right\rangle .
$$

The long-range scattering matrix is related to Green's function matrix by [18]

$$
\underline{S}^{\mathrm{LR}}=\frac{1}{i \pi}\left[\underline{f}^{-}\left(r_{0}\right)\right]^{-1} \underline{G}\left(r_{0}, r_{0}\right)\left[\underline{f}^{-}\left(r_{0}\right)\right]^{-1},
$$

where $r_{0}$ is the matching radius, $f^{-}$is the diagonal matrix

$$
f_{k k^{\prime}}^{-}(r)=f_{k}^{-}(r) \delta_{k k^{\prime}}
$$

comprising the radial wave functions, and $\underline{G}\left(r, r^{\prime}\right)$ denotes the part of $\widetilde{G}\left(r, r^{\prime}\right)$ satisfying incoming-wave boundary conditions at the final radius $r$. The latter condition ensures that only electron paths approaching the matching radius from the long-range region contribute to $\underline{S}^{\mathrm{LR}}$, whereas paths that traverse the core region are omitted.

\section{B. Closed-orbit theory for crossed-fields systems}

To obtain a semiclassical approximation to the long-range scattering matrix, we make use of the semiclassical Green's function derived by Gutzwiller [22]

$$
G^{\text {scl }}\left(\boldsymbol{x}, \boldsymbol{x}^{\prime} ; E\right)=\frac{2 \pi}{(2 \pi i)^{(n+1) / 2}} \sum_{\text {class. traj. }} \sqrt{|D|} \exp \left(i S-i \frac{\pi}{2} \sigma\right),
$$

where the sum extends over all classical trajectories leading from $\boldsymbol{x}^{\prime}$ to $\boldsymbol{x}$ at the energy $E, n$ is the number of degrees of freedom, $S$ is the classical action along the trajectory, $\sigma$ the number of caustics along the trajectory, and 


$$
D=\operatorname{det}\left(\begin{array}{cc}
\frac{\partial^{2} S}{\partial \boldsymbol{x} \partial \boldsymbol{x}^{\prime}} & \frac{\partial^{2} S}{\partial \boldsymbol{x} \partial E} \\
\frac{\partial^{2} S}{\partial E \partial \boldsymbol{x}^{\prime}} & \frac{\partial^{2} S}{\partial E^{2}}
\end{array}\right)
$$

is the amplitude for the contribution of the trajectory. By Eq. (14), we obtain a semiclassical approximation to Green's function matrix

$$
\begin{aligned}
G_{k k^{\prime}}^{\mathrm{scl}}\left(r_{0}, r_{0} ; E\right)= & \frac{2 \pi}{(2 \pi i)^{2}} r_{0}^{2} \int d \vartheta d \vartheta^{\prime} d \varphi d \varphi^{\prime} \sin \vartheta \\
& \times \sin \vartheta^{\prime} Y_{k}^{*}(\vartheta, \varphi) Y_{k^{\prime}}\left(\vartheta^{\prime}, \varphi^{\prime}\right) \\
& \times \sum_{\text {class. traj. }} \sqrt{|D|} e^{i\left(S\left(r_{0}, r_{0}\right)-\pi \sigma / 2\right)}
\end{aligned}
$$

As usual in semiclassics, the integrals will be evaluated in the stationary-phase approximation. It yields a sum over all classical trajectories, leaving the matching sphere at a direction given by $\left(\vartheta_{i}, \varphi_{i}\right)$ and returning to it at $\left(\vartheta_{f}, \varphi_{f}\right)$. The condition that $\underline{G}\left(r_{0}, r_{0}\right)$ obeys incoming-wave boundary conditions at the final radius translates into the condition that only orbits going out from the matching sphere into the longrange region and then returning to $r_{0}$ are to be included, whereas orbits passing through the core region are omitted. If all factors in the integrand except for the exponential are assumed to vary slowly, the stationary-phase approximation reads

$$
\begin{aligned}
G_{k k^{\prime}}^{\mathrm{scl}}\left(r_{0}, r_{0} ; E\right)= & 2 \pi r_{0}^{2} \sum_{\mathrm{i} \rightarrow \mathrm{f}} \sin \vartheta_{i} \sin \vartheta_{f} Y_{k}^{*}\left(\vartheta_{f}, \varphi_{f}\right) Y_{k^{\prime}}\left(\vartheta_{i}, \varphi_{i}\right) \\
& \times \frac{\sqrt{\left|D_{\text {s.p. }}\right|}}{\sqrt{\left|\operatorname{det} \frac{\partial^{2} S}{\partial\left(\vartheta^{\prime}, \varphi^{\prime}, \vartheta, \varphi\right)^{2} \mid}\right|}} \\
& \times \exp \left(i S\left(r_{0}, r_{0}\right)-i \frac{\pi}{2}(\sigma+\kappa)\right),
\end{aligned}
$$

where $\kappa$ is the number of negative eigenvalues of the Hessian matrix of $S$ occurring in the prefactor.

Because the initial state is assumed to be well localized, it is clear that the outgoing waves generated by the photoexcitation originate in the immediate neighborhood of the nucleus. Therefore, only trajectories leaving the matching sphere radially need to be included in Eq. (20). By the same token, the trajectories can be assumed to return to the matching radius radially. Thus, they are parts of closed orbits starting precisely at the nucleus and returning there.

By transforming (18) to spherical coordinates and making use of the relations

$$
\frac{\partial S}{\partial \boldsymbol{x}}=\boldsymbol{p}, \quad \frac{\partial S}{\partial E}=t
$$

the amplitude factor $D$ for radial trajectories can be simplified to

$$
D=-\frac{1}{\dot{r} \dot{r}^{\prime} r^{2} r^{\prime 2} \sin \vartheta \sin \vartheta^{\prime}} \operatorname{det} \frac{\partial\left(p_{\vartheta}^{\prime}, p_{\varphi}^{\prime}\right)}{\partial(\vartheta, \varphi)}
$$

The determinants occurring in Eq. (20) combine to

$$
\begin{aligned}
& \operatorname{det} \frac{\partial\left(p_{\vartheta}^{\prime}, p_{\varphi}^{\prime}\right)}{\partial(\vartheta, \varphi)}\left(\operatorname{det} \frac{\partial^{2} S}{\partial\left(\vartheta^{\prime}, \varphi^{\prime}, \vartheta, \varphi\right)^{2}}\right)^{-1} \\
& \quad=\operatorname{det} \frac{\partial\left(p_{\vartheta}^{\prime}, p_{\varphi}^{\prime}, p_{\vartheta}, p_{\varphi}\right)}{\partial\left(\vartheta, \varphi, p_{\vartheta}, p_{\varphi}\right)}\left(\operatorname{det} \frac{\partial\left(-p_{\vartheta}^{\prime},-p_{\varphi}^{\prime}, p_{\vartheta}, p_{\varphi}\right)}{\partial\left(\vartheta^{\prime}, \varphi^{\prime}, \vartheta, \varphi\right)}\right)^{-1} \\
& \quad=\operatorname{det} \frac{\partial\left(\vartheta^{\prime}, \varphi^{\prime}\right)}{\partial\left(p_{\vartheta}, p_{\varphi}\right)} .
\end{aligned}
$$

With these results, Green's function matrix assumes the form

$$
\begin{aligned}
& G_{k k^{\prime}}^{\mathrm{scl}}\left(r_{0}, r_{0} ; E\right)=2 \pi \sum_{\text {c.o. }} \frac{\sqrt{\sin \vartheta_{i} \sin \vartheta_{f}}}{\sqrt{\left|\dot{r} \dot{r}^{\prime}\right|}} \\
& \times \frac{Y_{k}^{*}\left(\vartheta_{f}, \varphi_{f}\right) Y_{k^{\prime}}\left(\vartheta_{i}, \varphi_{i}\right)}{\sqrt{\left|\operatorname{det} \frac{\partial\left(p_{\vartheta_{f}}, p_{\varphi_{f}}\right)}{\partial\left(\vartheta_{i}, \varphi_{i}\right)}\right|}} \\
& \times e^{i S\left(r_{0}, r_{0}\right)-i \pi(\sigma+\kappa) / 2} \text {. }
\end{aligned}
$$

The determinant in the denominator of Eq. (24) measures the dependence of the final angular momenta of the trajectory upon the starting angles. As it stands, it suffers from the singularities of the spherical coordinate chart: At the poles, neither the angle $\varphi$ nor the angular momenta $p_{\vartheta}$ and $p_{\varphi}$ are well defined, so that close to the poles, the calculation of the determinant becomes numerically unstable. The determinant can be rewritten in the form $[21,23]$

$$
\operatorname{det} \frac{\partial\left(p_{\vartheta_{f}}, p_{\varphi_{f}}\right)}{\partial\left(\vartheta_{i}, \varphi_{i}\right)}=\sin \vartheta_{i} \sin \vartheta_{f} M
$$

with a $2 \times 2$-determinant $M$ devoid of any singularities. The parameter $M$ was already used in Ref. [17] to study the bifurcations of closed orbits. We showed there that a closed orbit bifurcates if and only if $M=0$. With form (25) of the stability determinant, the semiclassical Green's function matrix reads

$$
\begin{aligned}
G_{k k^{\prime}}= & 2 \pi \sum_{\text {c.o. }} \frac{1}{\sqrt{\left|\dot{r} \dot{r}^{\prime}\right|}} \frac{Y_{k}^{*}\left(\vartheta_{f}, \varphi_{f}\right) Y_{k^{\prime}}\left(\vartheta_{i}, \varphi_{i}\right)}{\sqrt{|M|}} \\
& \times \exp \left(i S\left(r_{0}, r_{0}\right)-i \frac{\pi}{2}(\sigma+\kappa)\right),
\end{aligned}
$$


which is free of any singularities introduced by the spherical coordinates.

By virtue of Eq. (15), the semiclassical long-range scattering matrix reads

$$
\begin{aligned}
S_{k k^{\prime}}^{\mathrm{LR}}= & 2 i \sum_{\text {c.o. }} \frac{1}{\sqrt{\left|\dot{r} \dot{r}^{\prime}\right|}} \frac{1}{f_{k}^{-}\left(r_{0}\right)} \frac{1}{f_{k^{\prime}}^{-}\left(r_{0}\right)} \\
& \times \frac{Y_{k}^{*}\left(\vartheta_{f}, \varphi_{f}\right) Y_{k^{\prime}}\left(\vartheta_{i}, \varphi_{i}\right)}{\sqrt{|M|}} e^{i S\left(r_{0}, r_{0}\right)-i \pi(\sigma+\kappa) / 2} .
\end{aligned}
$$

This expression can be further simplified if, for excited states close to the ionization threshold, the radial wave functions

$$
f_{l}^{-}(r) \approx-i \sqrt{r} H_{2 l+1}^{(2)}(\sqrt{8 r})
$$

are approximated by the zero-energy wave functions, and the Hankel functions are replaced with their asymptotic forms for large arguments [24]:

$$
H_{\nu}^{(2)}(x) \approx \sqrt{\frac{2}{\pi x}} \exp \left(-i x+i \frac{\pi}{2} \nu+i \frac{\pi}{4}\right) .
$$

This approximation leads to

$$
\begin{aligned}
S_{l m, l^{\prime} m^{\prime}}^{\mathrm{LR}}= & -2 \pi \sum_{\text {c.o. }}(-1)^{l+l^{\prime}} \frac{Y_{l m}^{*}\left(\vartheta_{f}, \varphi_{f}\right) Y_{l^{\prime} m^{\prime}}\left(\vartheta_{i}, \varphi_{i}\right)}{\sqrt{|M|}} \\
& \times \exp \left(i\left[S\left(r_{0}, r_{0}\right)+2 \sqrt{8 r_{0}}\right]-i \frac{\pi}{2}(\sigma+\kappa)\right),
\end{aligned}
$$

because, due to the conservation of energy, $\dot{r}^{2} / 2=1 / r$ if $E$ $=0$. In Eq. (30), the channel indices $k=(l, m)$ are finally written out explicitly.

For a radial trajectory in a hydrogen atom going out from the nucleus to $r=r_{0}$ at zero energy, the action is $\sqrt{8 r_{0}}$, so that

$$
S_{\text {c.o. }}=S\left(r_{0}, r_{0}\right)+2 \sqrt{8 r_{0}}
$$

is the action of a closed orbit, measured from its start at the nucleus to its return. The semiclassical long-range $S$ matrix finally reads

$$
\begin{aligned}
S_{l m, l^{\prime} m^{\prime}}^{\mathrm{LR}}= & -2 \pi \sum_{\text {c.o. }}(-1)^{l+l^{\prime}} \frac{Y_{l m}^{*}\left(\vartheta_{f}, \varphi_{f}\right) Y_{l^{\prime} m^{\prime}}\left(\vartheta_{i}, \varphi_{i}\right)}{\sqrt{|M|}} \\
& \times \exp \left(i S_{\text {c.o. }}-i \frac{\pi}{2}(\sigma+\kappa)\right) .
\end{aligned}
$$

Both the action $S_{\text {c.o. }}$ and the stability determinant $M$ are evaluated at the nucleus rather than on the matching sphere. The response function is given by

$$
g^{\mathrm{osc}}(E)=4 \pi \sum_{\text {c.o. }} \frac{\mathcal{Y}^{*}\left(\vartheta_{f}, \varphi_{f}\right) \mathcal{Y}\left(\vartheta_{i}, \varphi_{i}\right)}{\sqrt{|M|}} \exp \left(i S_{\text {c.o. }}-i \frac{\pi}{2} \mu\right) \text {, }
$$

where the Maslov index $\mu=\sigma+\kappa+1$ was increased by 1 to absorb an additional phase, and the function

$$
\mathcal{Y}(\vartheta, \varphi)=\sum_{l m}(-1)^{l} d_{l m} Y_{l m}(\vartheta, \varphi)
$$

with the core-region matrix elements $d_{l m}$ given by Eq. (10), characterizes the initial state and the exciting photon. Through $d_{l m}$, the function $\mathcal{Y}(\vartheta, \varphi)$ is energy dependent. In accordance with the choice of zero-energy radial wave functions in the $S$-matrix elements, $\mathcal{Y}(\vartheta, \varphi)$ will be evaluated at zero energy. This approximation has proven accurate in all applications of closed-orbit theory considered in the literature so far. However, from the $S$-matrix theory derivation it is obvious that the energy dependence of both the dipole matrix elements $d_{l m}$ and the $S$-matrix elements can easily be included should the need arise. The semiclassical response function (33) has anticipated form (3) with

$$
\mathcal{A}_{\text {c.o. }}=4 \pi \frac{\mathcal{Y}^{*}\left(\vartheta_{f}, \varphi_{f}\right) \mathcal{Y}\left(\vartheta_{i}, \varphi_{i}\right)}{\sqrt{|M|}} e^{i(\pi / 2) \mu} .
$$

\section{THE SCALED QUANTUM SPECTRUM}

If Schrödinger's equation for the crossed-fields hydrogen atom is rewritten in terms of the scaled energy and the scaled electric field strength, a quadratic eigenvalue problem for the scaling parameter $w$ is obtained. An exact numerical method of solution for the quadratic eigenvalue problem has become available only recently [25]. We resort to the method introduced by Main [15], which relies on an approximate linearization of the eigenvalue problem to compute eigenvalues in a small spectral interval. The accuracy of the linearization can be verified by comparing results that were calculated using different overlapping intervals. The eigenvalues are obtained to a relative accuracy of at least $10^{-7}$, which is far beyond the typical accuracy of semiclassical approximations, so that the algorithm is well suited to this study.

In the following we will discuss quantum and semiclassical photoabsorption spectra obtained for the scaled energy $\widetilde{E}=-1.4$ and the scaled electric field strength $\widetilde{F}=0.1$ with the initial state $|2 p 0\rangle$ and light linearly polarized along the magnetic field axis. A quantum spectrum for these parameter values is shown in Fig. 1. As for a semiclassical analysis (see Sec. VII) it is essential to have as many eigenvalues available as possible, the calculation was extended up to $w$ $=100$. The spectrum shown in Fig. 1 contains nearly 30000 lines, many of which are too weak to be discernible in the plot.

The eigenenergies of the field-free hydrogen atom satisfy

$$
E=w^{-2} \widetilde{E}=-\frac{1}{2 n^{2}}
$$



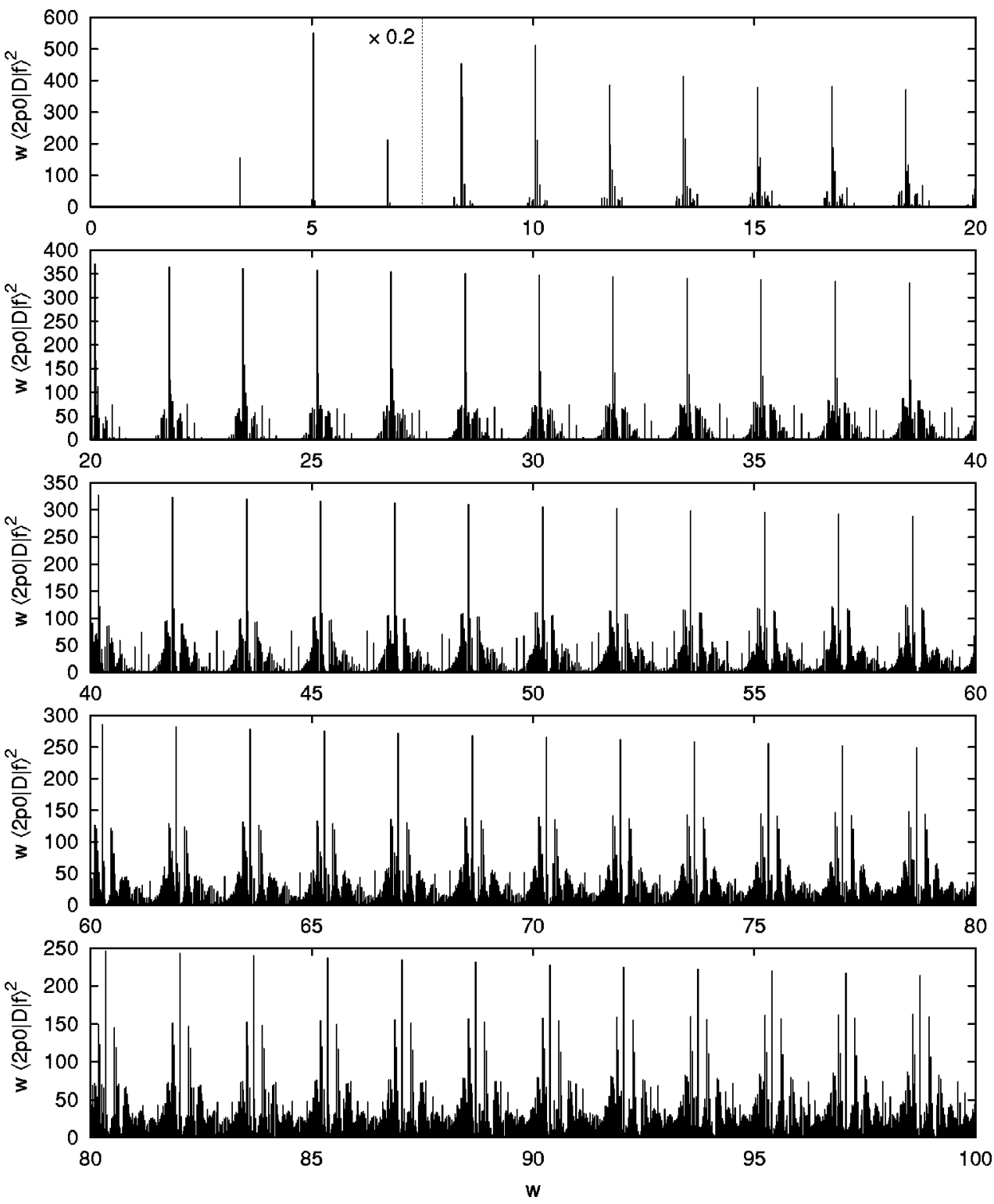

FIG. 1. Quantum photoabsorption spectrum at the scaled energy $\widetilde{E}=-1.4$ and the scaled electric field strength $\widetilde{F}=0.1$. The initial state is $|2 p 0\rangle$, the light is polarized along the magnetic-field axis. The plot shows the squared dipole matrix elements, which for graphical reasons are multiplied by $w$. The strengths of the extraordinarily strong lines of the lowest $n$ manifolds at $w<7.5$ are scaled down by a factor of 0.2 .

so that in the scaled spectrum the unperturbed $n$ manifolds appear equidistantly spaced at

$$
w=\sqrt{-2 \widetilde{E}} n .
$$

These spacings can clearly be recognized in Fig. 1. At low values of $w$, neighboring $n$ manifolds are isolated. Furthermore, in this region the magnetic quantum number $m$ is nearly conserved. This is apparent from the fact that each $n$ manifold contains a central group of strong levels corresponding to $m=0$, which can be excited even at $\widetilde{F}=0$, and adjacent groups of considerably weaker levels with $m$ $= \pm 1$. Levels with higher magnetic quantum numbers are too weak in this region to be seen in the figure. At higher values of $w$, the conservation of $m$ is violated, and individual $n$ manifolds acquire strong side bands. At even higher $w$, different $n$ manifolds strongly overlap. Throughout the spectral range shown, groups of strong lines indicating the centers of different $n$ manifolds are clearly discernible.

\section{LOW-RESOLUTION SEMICLASSICAL SPECTRA}

A semiclassical approximation to a scaled photoabsorption spectrum is obtained if the closed-orbit theory formulas of Sec. III B are rewritten in terms of scaled quantities, viz.

$$
g^{\mathrm{osc}}(w)=\frac{1}{w} \sum_{\text {c.o. }} \widetilde{\mathcal{A}}_{\text {c.o. }} \exp \left(i w \widetilde{S}_{\text {c.o. }}\right)
$$

with 


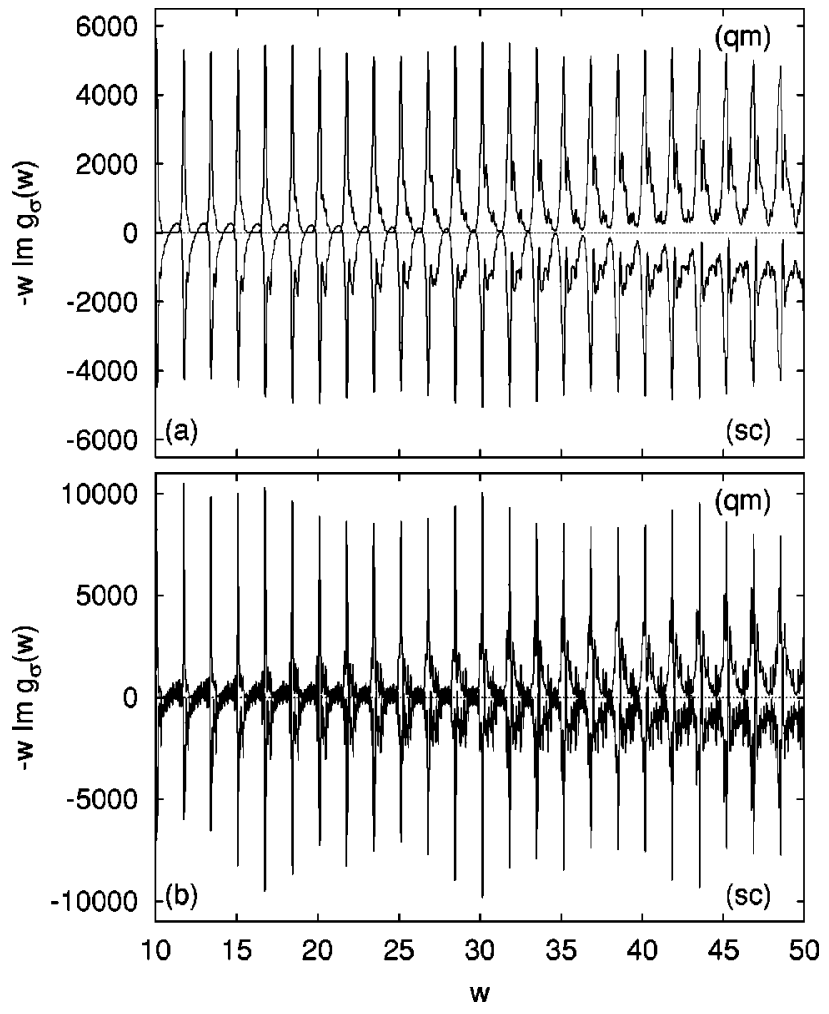

FIG. 2. Smoothed quantum (upper halves) and semiclassical (lower halves, inverted) photoabsorption spectra with cut-off action (a) $\sigma=20$ and (b) $\sigma=50$.

$$
\widetilde{\mathcal{A}}_{\text {c.o. }}=4 \pi \frac{\mathcal{Y}^{*}\left(\vartheta_{f}, \varphi_{f}\right) \mathcal{Y}\left(\vartheta_{i}, \varphi_{i}\right)}{\sqrt{|\tilde{M}|}} e^{i(\pi / 2) \mu} .
$$

When low-resolution photoabsorption spectra are to be calculated from Eq. (38), a method of cutoff must be adopted to deal with the divergence of the semiclassical closed-orbit sum. For this section, we choose a Gaussian cutoff, i.e., Eq. (38) is replaced with

$$
g_{\sigma}^{\mathrm{osc}}(w)=\frac{1}{w} \sum_{\text {c.o. }} \widetilde{\mathcal{A}}_{\text {c.o. }} \exp \left(i w \widetilde{S}_{\text {c.o. }}-\frac{\widetilde{S}_{\text {c.o. }}^{2}}{2 \sigma^{2}}\right),
$$

so that orbits with scaled actions larger than the cutoff action $\sigma$ are smoothly suppressed. This smoothing corresponds to a convolution of the quantum signal with a Gaussian of width $1 / \sigma$.

To facilitate the comparison of Eq. (40) with the convoluted quantum spectrum, we added the smooth part of the spectrum to $g_{\sigma}^{\text {osc }}$, which was calculated by convoluting the quantum spectrum with a Gaussian of width $1 / \sigma=1$. This function is broad enough to wipe out the distinction between neighboring principal quantum numbers. Results obtained for $\sigma=20$ and $\sigma=50$ are shown in Fig. 2. In both cases it is apparent that the large-scale structure of equidistant principal quantum numbers is well reproduced by the semiclassical approximation. In the quantum spectra, the substructure of the individual $n$ shells can be discerned to a certain degree, given by the smoothing width $1 / \sigma$. In the case of $\sigma=20$, much of this fine structure is also present in the semiclassical spectrum, but often the agreement is not good quantitatively. In particular, the peaks corresponding to the lowest $n$-manifolds are considerably wider in the semiclassical than in the quantum spectrum.

If the cutoff action is increased to $\sigma=50$, finer details are resolved in the quantum spectrum. At the same time, the semiclassical closed-orbit sum becomes more oscillatory to reproduce this fine structure. It appears, however, to be somewhat overoscillatory, developing structures absent from the quantum spectrum. This type of behavior is typical of closed-orbit sums in nonintegrable systems. Thus, it can be questioned if the low-resolution closed-orbit sum can meaningfully be extended to even longer orbits. A high-resolution quantization based on the present semiclassical approximation will be presented in the following section.

\section{HIGH-RESOLUTION SEMICLASSICAL SPECTRA}

For the calculation of a scaled semiclassical spectrum, the method of semiclassical quantization by harmonic inversion of $\delta$ function signals $[14,26]$ can be applied. This technique requires the inclusion of closed orbits up to a maximum scaled action, i.e., it replaces the Gaussian cutoff used for the low-resolution semiclassical spectra presented in the preceding section with a rectangular cutoff. A rough estimate for the required cutoff action can be obtained by means of perturbation theory [21]:

$$
\widetilde{S}_{\max }=-8 \pi \widetilde{E} n
$$

For the case $\widetilde{E}=-1.4$ and $n=9$, i.e., $w=15.06$, this estimate yields $\widetilde{S}_{\max } / 2 \pi \approx 50$.

According to Eq. (41), to compute levels at high quantum numbers $n$ a long semiclassical signal is needed, which can be hard or even impossible to obtain. We calculated closed orbits up to $\widetilde{S}_{\max } / 2 \pi=200$, so that the orbital data is available for nearly 18000 closed-orbit multiplets. However, for reasons to be described in Sec. VII a useful semiclassical signal can be constructed up to $\widetilde{S}_{\max } / 2 \pi \approx 60-70$ only, so that from the above estimate, the semiclassical calculation cannot reach manifolds much higher than $n=10$. On the other hand, the semiclassical approximation must be expected to yield more accurate results for higher quantum numbers. Thus, when a high-resolution semiclassical spectrum is to be calculated, a compromise must be made between the contradictory requirements of describing a spectral region at sufficiently high quantum numbers and with a sufficiently low spectral density. For the harmonic analysis of the closed-orbit sum we applied the method of $\delta$ function decimated signal diagonalization [26,27], which yields not only semiclassical eigenvalues and amplitudes, but also an error parameter estimating the precision of the eigenvalues. Results obtained for $\widetilde{E}=-1.4$ and $\widetilde{F}=0.1$ with a signal length of $\widetilde{S}_{\max } / 2 \pi=60$ are compiled in Table I. The table contains the quantum eigenvalues of $w$ and their dipole matrix elements for the levels satisfying $\langle 2 p 0|D| f\rangle^{2}>0.7$. It is obvious at a glance that out of the multitude of spectral lines 
TABLE I. Semiclassical and quantum eigenvalues $w_{f}$ of the scaling parameter for $\widetilde{E}=-1.4$ and $\widetilde{F}=0.1$. See text for a detailed description. The dipole matrix elements $\langle 2 p 0|D| f\rangle^{2}$ were obtained from a quantum spectrum.

\begin{tabular}{|c|c|c|c|c|c|c|c|}
\hline$n$ & $w_{f}(\mathrm{scl})$. & $w_{f}(\mathrm{qm})$. & $\langle 2 p 0|D| f\rangle^{2}$ & $n$ & $w_{f}(\mathrm{scl})$. & $w_{f}$ (qm.) & $\langle 2 p 0|D| f\rangle^{2}$ \\
\hline & & 9.88321 & 1.3617 & & 15.12905 & 15.12748 & 10.3140 \\
\hline & & 9.97747 & 1.7474 & & 15.23623 & 15.23830 & 3.1064 \\
\hline & 10.05366 & 10.05912 & 51.0512 & & 15.26111 & 15.27005 & 1.7749 \\
\hline \multirow[t]{6}{*}{6} & 10.09551 & 10.09621 & 20.9313 & & & 15.30024 & 2.3710 \\
\hline & & 10.24076 & 0.9608 & & & 15.40389 & 3.3462 \\
\hline & & 10.26612 & 2.0777 & & & & \\
\hline & & 10.31803 & 1.9385 & & & 16.57908 & 0.7173 \\
\hline & & & & & & 16.58435 & 1.7007 \\
\hline & & 11.56497 & 2.5663 & & & 16.60357 & 1.7437 \\
\hline \multirow{10}{*}{7} & & 11.78850 & 10.0092 & 10 & 16.78346 & 16.78269 & 11.1809 \\
\hline & & 11.84856 & 5.6249 & & 16.81329 & 16.81827 & 6.6898 \\
\hline & & 11.92188 & 1.9229 & & & 16.86870 & 0.9825 \\
\hline & & 11.95821 & 1.7923 & & 16.93431 & 16.93323 & 2.0584 \\
\hline & & 12.01338 & 2.4821 & & & 16.94303 & 1.4143 \\
\hline & & & & & & 16.96000 & 1.4406 \\
\hline & & 13.23441 & 1.3668 & & & 16.99085 & 2.3893 \\
\hline & & 13.25629 & 2.5141 & & & 17.09909 & 3.5870 \\
\hline & & 13.30255 & 1.9971 & & & 17.25847 & 0.7647 \\
\hline & 13.36921 & 13.36913 & 2.8189 & & & & \\
\hline \multirow{5}{*}{8} & & 13.70866 & 2.9676 & 11 & 18.47472 & 18.47149 & 7.2231 \\
\hline & & & & & & 18.50996 & 4.0510 \\
\hline & & 14.91192 & 2.1880 & & & 18.61835 & 1.7975 \\
\hline & & 14.94654 & 2.9922 & & & 18.62818 & 1.2089 \\
\hline & & 14.99711 & 1.4563 & & & 18.64563 & 2.2348 \\
\hline \multirow[t]{3}{*}{9} & 15.06960 & 15.06470 & 3.2226 & & & 18.68226 & 2.2558 \\
\hline & & 15.07888 & 25.1866 & & & 18.79427 & 3.6707 \\
\hline & & 15.10074 & 8.4317 & & 18.93585 & 18.95442 & 1.0263 \\
\hline
\end{tabular}

with intensities varying over many orders of magnitude (most of which are not contained in the table) only the strongest lines were detected in the semiclassical spectrum. The semiclassical eigenvalues given are characterized by having small imaginary parts, small error parameters, and large amplitudes as well as being stable with respect to a variation of numerical parameters. The calculation operates at the edge of convergence, and in a few cases one can be in doubt whether a level should be included according to these fairly "soft" criteria, but in general a clear decision can be made. Semi- classical values for the transition strengths are not given because they are not reasonably well converged and depend strongly on the numerical parameters.

One might expect that in each $n$ manifold the strongest lines are detected semiclassically, and in general this expectation is confirmed by the numerical data. This can clearly be seen, e.g., in the manifold $n=6$, which contains the most stably converged lines in the spectrum. There are, however, a few conspicuous exceptions, e.g., at $n=7$, where strong lines are missing, whereas comparatively weak lines are 
found. For $n=5$, no lines at all can be computed from the given semiclassical signal. If the signal length is decreased to $\widetilde{S}_{\max } / 2 \pi=50$, the three strongest lines appear in the spectrum in this manifold.

At higher $n$, the number of strong lines in the quantum spectrum increases. So does the number of lines in the semiclassical spectrum until $n=11$, where only three semiclassical lines are found. They appear rather arbitrarily scattered across the quantum spectrum, and their convergence is notably worse than in lower manifolds. It is clear that in this $n$ shell the semiclassical quantization with the given signal is about to break down. At $n=12$, no lines can be detected semiclassically. As, from the above discussion, this failure was to be expected because the required signal length becomes too large, the obvious way to improve convergence seems to be to use a longer signal. However, if the signal length is increased to $\widetilde{S}_{\max } / 2 \pi=70$, no reasonably converged semiclassical lines can be found in any $n$ manifold. Neither are results improved if the technique of harmonic inversion of cross-correlated closed-orbit sums [16,28] is applied. This method has proven powerful in reducing the signal length required in a semiclassical quantization. In the present case, however, because the cross correlation increases the total number of frequencies obtained from the harmonic inversion, the true eigenvalues are hidden among a multitude of spurious frequencies, and no useful results can be obtained.

For the time being, therefore, the results given in Table I represent what can be achieved in the semiclassical quantization of the crossed-fields hydrogen atom. They confirm the applicability of the closed-orbit theory approach, in principle, but they also reveal a fundamental problem in its present formulation. It is clear that the signal length available is sufficient for a stable signal analysis. Thus, if the semiclassical results are not good, the semiclassical signal itself, rather than the signal analysis, must be to blame. This conclusion is confirmed by the observation that an increased signal length destroys the results rather than improves them. We therefore start searching for a flaw in the construction of the semiclassical photoabsorption spectrum.

A conspicuous problem lies in the fact that the set of closed orbits available is incomplete. In no series of rotators or vibrators can arbitrarily long orbits be calculated. In the case of vanishing electric field, there is a critical angle $\vartheta_{\mathrm{c}}$ that the starting angles of both rotators and vibrators approach as the orbits get longer. This convergence indicates that the orbits approach a separatrix between two families of tori in phase space. If sufficiently long orbits are studied, there are many closed orbits with very similar initial conditions, so that the numerical search for closed orbits must eventually fail.

The region of phase space where the unknown orbits are located is lying close to a separatrix, so that it is highly unstable. The orbits can therefore be expected not to contribute much to the semiclassical signal. The magnitude of an orbit's contribution to the closed-orbit sum (33) is determined mainly by its stability determinant $M$. Figure 3 shows the stability determinants of the vibrator orbits for $\widetilde{E}$ $=-1.4, \widetilde{F}=0.1$ as a function of the scaled action. Different

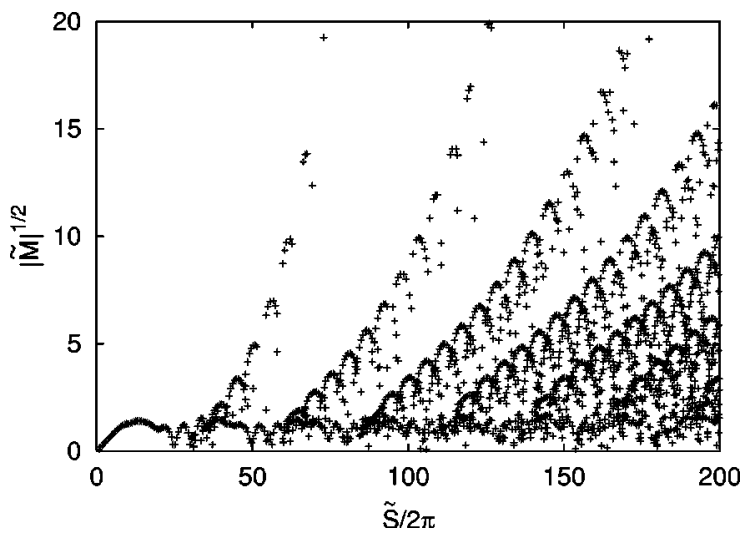

FIG. 3. Stability determinants of vibrators as a function of the action for $\widetilde{E}=-1.4, \widetilde{F}=0.1$.

series of vibrators can clearly be discerned in the plot. It is indeed unstable orbits with large $\widetilde{M}$ that are missing in the data set, but on the other hand the stability determinants of the missing orbits are not large enough to regard the corresponding semiclassical amplitudes as negligibly small. Because a vast majority of orbits has small $\widetilde{M}$ and was found, one can still hope that useful results can be obtained from the semiclassical signal, at least for quantum states not located in the separatrix region in phase space, but it is clear that the quality of the semiclassical signal is reduced by its incompleteness.

To assess in detail the detrimental effect of the missing orbits and of any other sources of error that may exist, we carry out a semiclassical analysis of the quantum spectrum.

\section{SEMICLASSICAL RECURRENCE SPECTRA}

According to Eq. (38), in a scaled photoabsorption spectrum every closed orbit contributes a purely sinusoidal modulation to $w g(w)$. This contribution can be extracted from the spectrum either by a conventional Fourier transform or by means of a high-resolution method. The spectral analysis yields information about classical orbits returning to the nucleus. For this reason, the transformed spectrum is referred to as a recurrence spectrum. High-resolution methods [15] extract the scaled actions and scaled semiclassical amplitudes of individual orbits and thus yield more complete information about the semiclassical spectrum than the Fourier transform, but they fail if the average density of closed orbits per unit of scaled action is too large. By contrast, due to its linearity the Fourier transform can be applied to any part of the recurrence spectrum with equal ease and numerical stability, irrespective of the spectral density. In dense regions, it will not be able to identify individual closed orbits, but it will nevertheless yield a recurrence spectrum that can be compared to the classical data. In this section we will present results obtained by both the Fourier transform and a highresolution method. The semiclassical recurrence spectra will be compared to classical results in order to identify the reason why the semiclassical signal is only partially suitable to a semiclassical quantization.

Using either method, it is essential to note that the semi- 

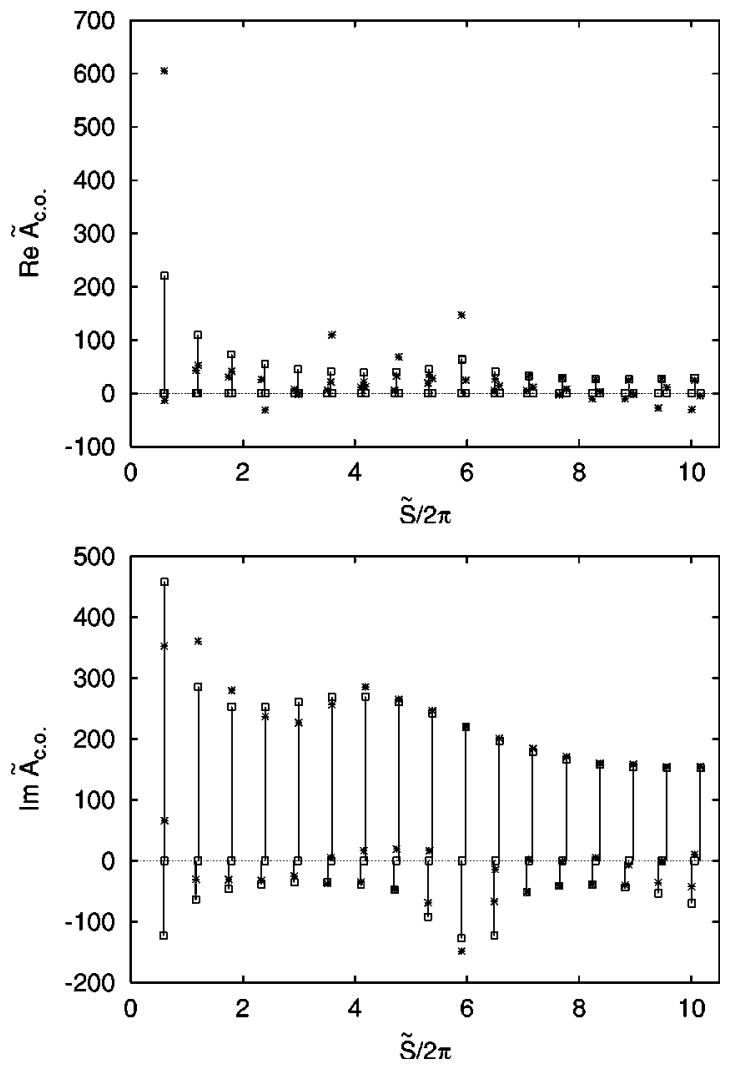

FIG. 4. High-resolution recurrence spectrum for $\widetilde{E}=-1.4$ and $\widetilde{F}=0.1$. Sticks and squares: semiclassical closed-orbit amplitudes. Stars: harmonic inversion of the quantum spectrum.

classical closed-orbit formula cannot be expected to yield accurate results for the lowest levels. Thus, the low $n$ manifolds must be excluded from the semiclassical analysis, i.e., the analysis is based on the quantum spectrum given in an interval $\left[w_{\min }, w_{\max }\right]$ instead of $\left[0, w_{\max }\right]$. Furthermore, to minimize the impact of boundary effects due to the finite length of the semiclassical spectrum, a smooth Gaussian cutoff with width $\kappa$ centered at $w_{0}=\left(w_{\min }+w_{\max }\right) / 2$ is introduced. The smoothing replaces the peaks of the semiclassical recurrence spectrum by Gaussians of width $1 / \kappa$. The recurrence spectra presented here were calculated from the quantum spectrum shown in Fig. 1 , for $\widetilde{E}=-1.4$ and $\widetilde{F}=0.1$, with $w_{\min }=20, w_{\max }=100$, and $\kappa=10$. For the highresolution recurrence spectra, the method of $\delta$ function decimated signal diagonalization was used.

For low-scaled actions, where only a few closed orbits exist, the high-resolution analysis can be applied. Results are shown in Fig. 4, which compares both the scaled actions and the real and imaginary parts of the semiclassical amplitudes extracted from the quantum spectrum to the classical results. For most closed orbits, the agreement is excellent. Exceptions occur for the shortest orbits, where the actions of rotator and vibrator orbits are too similar to be resolved by the harmonic inversion. At somewhat larger actions, the three orbits in each group fall apart into two rotator orbits with similar actions and a vibrator orbit with a slightly larger action.

These observations can be made even more clearly if the
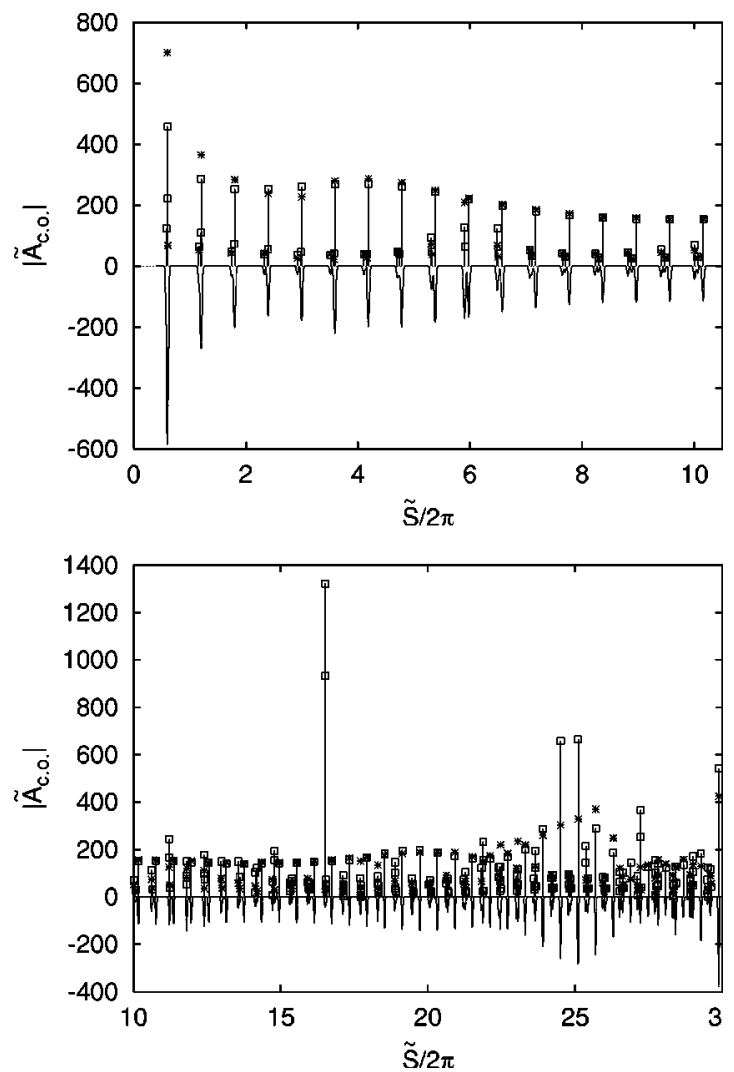

FIG. 5. Absolute value of the recurrence spectrum. Sticks and squares: semiclassical closed-orbit amplitudes. Stars: harmonic inversion of the quantum spectrum. Solid curve, inverted: Fourier transform (arbitrary units).

absolute values of the amplitudes are considered. They are shown in Fig. 5, where the results of the high-resolution analysis are also compared to those of the Fourier transform. Notice that for the Fourier transform the semiclassical amplitude is given by the area under a peak rather than the peak height, so that an immediate comparison to the highresolution results is difficult. In Fig. 5, the Fourier transform was arbitrarily scaled, so that the peak heights roughly match the values of the high-resolution amplitudes. For isolated orbits identified both in the Fourier transform and the highresolution spectrum, the agreement between the two methods is excellent. Where several peaks overlap in the semiclassical spectrum, no direct comparison is possible because the peak phases cannot be determined from the figure.

Figure 5 also extends the results shown in Fig. 4 to higher actions. In this region the density of closed orbits starts to increase because, on the one hand, rotators of the first series exist and, on the other, bifurcations of closed orbits generate additional orbits. Apart from the fact that many orbits cannot be identified individually even by the high-resolution method, the most conspicuous feature of Fig. 5 is that for many orbits the semiclassical amplitudes calculated from the classical data are considerably larger than those extracted from the quantum spectrum. In some cases, this is obvious at a glance, but a closer inspection of the figure reveals that this phenomenon is rather common. Some specific cases will be described in detail in Sec. VIII. 

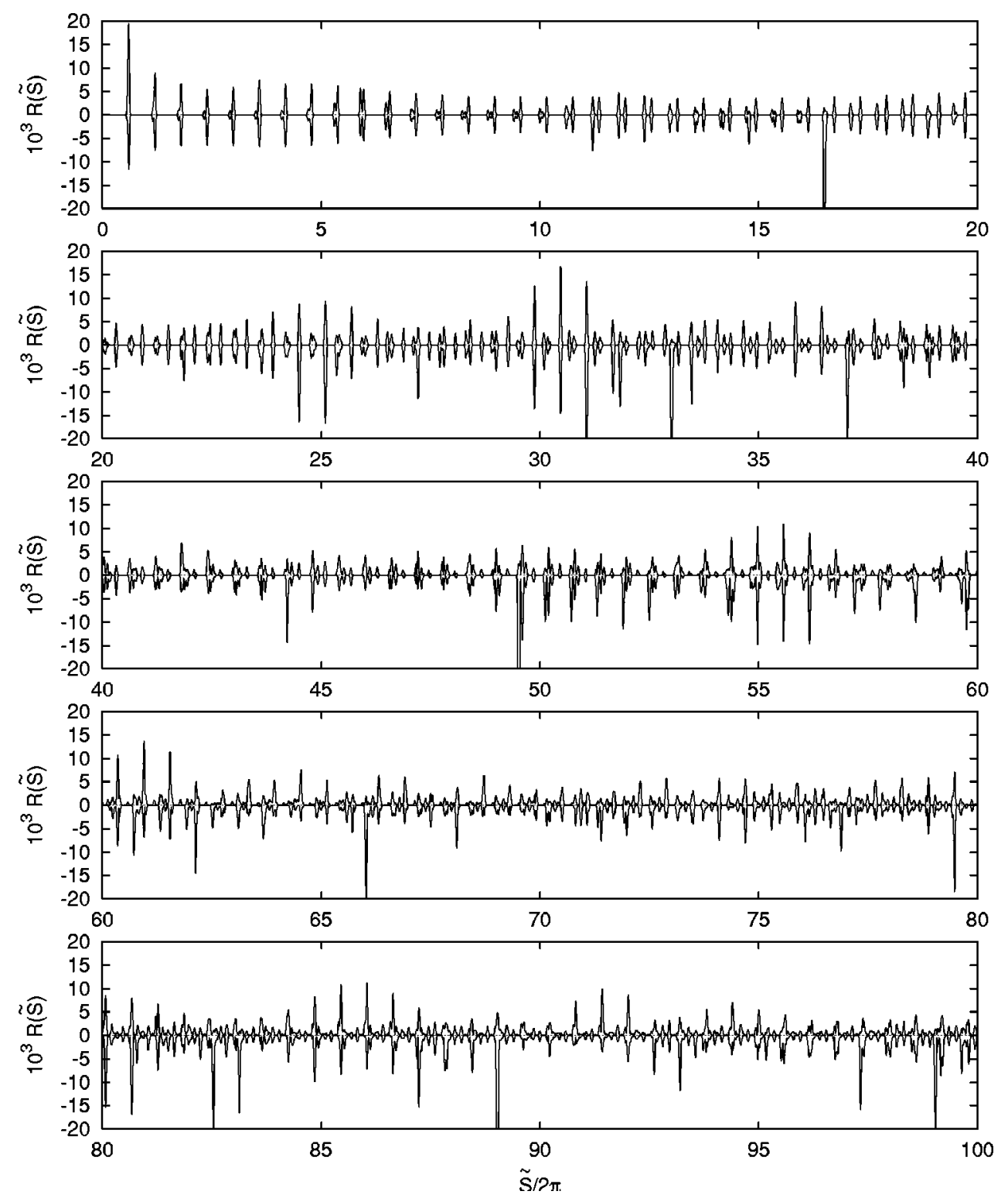

FIG. 6. Absolute value $R(\widetilde{S})$ of the recurrence spectrum with $\kappa=10$ (see text). Upper part: Fourier transform of the quantum spectrum. Lower part (inverted): smoothed semiclassical recurrence spectrum.

The occurrence of exceedingly large semiclassical amplitudes is a well-known problem of both closed-orbit and periodic-orbit theory. It is associated with bifurcations of classical orbits, where, in the case of closed orbits, the stability determinant $M$ vanishes and the closed-orbit amplitude (35) diverges. Close to the bifurcation, $M$ is small. The semiclassical amplitude of the bifurcating orbit is therefore large and exceeds the value determined from the quantum spectrum. In a classical context, we have shown previously [17] that vanishing $M$ is both a necessary and sufficient condition for a bifurcation of closed orbits. In the context of semiclassical closed-orbit theory, it is necessary to overcome the divergence of the closed-orbit formula occurring close to a bifurcation. This problem will be addressed in Sec. VIII, after the impact of the bifurcations on the semiclassical signal at hand has been investigated further.

Although, in Fig. 5, the vibrator orbits are sufficiently isolated to be resolved by both the harmonic inversion and the Fourier transform across the entire range of actions, the rotators occur in groups of several orbits having nearly identical actions. They are not resolved properly by either method. Instead, the Fourier transform produces peaks describing the collective contribution of the orbits in a group. The harmonic inversion fits this contribution with fewer actions and amplitudes than the actual number of orbits. Although the results can be expected to reproduce the quantum spectrum fairly well, the principal virtue of the highresolution analysis - that it is capable of giving individual rather than collective contributions-is lost. It is therefore pointless to extend the high-resolution analysis to higher actions unless a significantly longer quantum spectrum can be obtained, and only the Fourier transform will be used henceforth.

Figure 6 displays the Fourier recurrence spectrum with 
smoothing $\kappa=10$ for scaled actions up to $\widetilde{S} / 2 \pi=100$ and compares it to the semiclassical spectrum. These results extend the semiclassical analysis of quantum spectra to significantly longer orbits than investigated in previous studies. They allow a verification of the closed-orbit theory all the way up to the long orbits. It is immediately apparent from the figure that the quantum recurrence spectrum retains its pronounced peak structure. This is to be expected from the closed-orbit theory, and indeed the peak locations are given by the actions of closed orbits for long as well as for short orbits. The basic idea of the closed-orbit theory that recurrence peaks are related to classical closed orbits is therefore confirmed in principle even for very long orbits.

Even for the largest actions considered, the quantum and semiclassical recurrence spectra agree quantitatively for some peaks. For most peaks, however, the peak heights in the quantum and semiclassical spectra disagree. There are quantum peaks that are smaller in the semiclassical spectrum or even completely absent. They can be attributed to missing orbits. On the other hand, in many cases the semiclassical peaks are significantly higher than the quantum peaks, sometimes by several orders of magnitude. Exceedingly high peaks can be traced back to bifurcations of closed orbits if the possibility is ignored that a quantum peak can be small because orbits missing in the semiclassical spectrum interfere destructively with the orbits present. This latter mechanism becomes the more implausible the larger the semiclassical peak is in comparison to the quantum peak.

Taken together, the effects of missing orbits and of bifurcating orbits distort the semiclassical recurrence spectrum to the point where it can no longer be expected to provide a suitable basis for a quantization. A close inspection of the recurrence spectrum suggests that the problem posed by bifurcating orbits is more severe. Exceedingly high peaks occur frequently. In addition, the very fact that they are high increases their detrimental effect on the semiclassical photoabsorption spectrum. Unless a suitable scheme for dealing with bifurcating orbits can be devised, no improvement of the semiclassical signal can be expected. We therefore turn to a description of the semiclassical treatment of bifurcating orbits by means of uniform approximations.

\section{UNIFORM APPROXIMATIONS}

\section{A. The construction of uniform approximations}

Exceedingly large contributions of single orbits to a semiclassical spectrum arise when the orbits are too close to a bifurcation to be regarded as isolated, as is implicitly assumed by the stationary-phase approximation used in the derivation of the closed-orbit formula. Uniform approximations that furnish a collective contribution of several nearly coincident trajectories were developed in the context of the semiclassical theory of molecular collisions (see, e.g., Refs. $[29,30])$. They were introduced in the periodic-orbit theory by Ozorio de Almeida and Hannay [31]. Their original approach was extended by different authors [32-35], so that today uniform approximations are a well-established tool of semiclassical physics. In Ref. [17], we identified two types of generic closed-orbit bifurcations of codimension 1. The pertinent uniform semiclassical approximations will be derived in what follows.

In most cases of interest, a bifurcation destroys real orbits and turns them into complex ghost orbits that exist in the complexified classical phase space. Ghost orbits can yield palpable contributions to semiclassical spectra $[35,36]$. In particular, their knowledge is essential for the construction of uniform approximation. For the generic closed-orbit bifurcations, the ghost orbits were described along with the real orbits in Ref. [17].

Of particular importance is the observation that bifurcations of codimension higher than 1 are relevant to semiclassics, although on a classical level they are not generically encountered. They appear as sequences of generic bifurcations, which, if the individual bifurcations are sufficiently close, must be described collectively by a single uniform approximation. Several examples of uniform approximations for these complicated bifurcation scenarios have been described in the literature [35,37-39].

The principal requirement a uniform approximation must satisfy is to asymptotically reproduce the known isolatedorbits approximation when the distance from the bifurcation grows large, because in this limit the stationary-phase approximation can be expected to be accurate. In the following, we will advocate a somewhat heuristic technique for the construction of a uniform approximation, which is easy to handle and yields a smooth interpolation between the asymptotic isolated-orbits approximations on either side of the bifurcation. It will first be described in general terms. Subsequently, uniform approximations describing the generic types of codimension-1 bifurcations of closed orbits will be derived.

A bifurcation scenario is described by a normal form $\Phi_{a}(t)$ depending on $n \geqslant 1$ variables $t$ and $m \geqslant 1$ parameters $a$, such that for any fixed value of the parameters $a$ there are stationary points of $\Phi_{a}(t)$ corresponding to the closed orbits involved in the bifurcation. The parameters $a$ must then depend on the energy $E$ to reproduce the bifurcations of the closed orbits.

For the uniform approximation we make the ansatz

$$
\Psi(E)=I(a) e^{i S_{0}(E)}
$$

with

$$
I(a)=\int_{\mathbb{R}^{n}} d^{n} t p(t) e^{i \Phi_{a}(t)} .
$$

Here, the functions $S_{0}(E)$ and $p(t)$ as well as the parameter values $a(E)$ have to be determined. All of them must be smooth functions of $E$.

To find the asymptotic behavior of uniform approximation (42) far from the bifurcations, Eq. (43) is evaluated in the stationary-phase approximation, which yields

$$
\Psi(E) \approx \sum_{t_{i}} \frac{(2 \pi i)^{n / 2} p\left(t_{i}\right)}{\sqrt{\left|\mathfrak{H} \Phi_{a}\left(t_{i}\right)\right|}} e^{i\left[S_{0}(E)+\Phi_{a}\left(t_{i}\right)\right]} e^{-i \pi \nu_{i} / 2},
$$


where the sum extends over all stationary points $t_{i}$ of $\Phi_{a}(t)$ that are real at the given $a, \mathfrak{H} \Phi_{a}$ is the Hessian determinant of $\Phi_{a}$, and $\nu_{i}$ is the number of negative eigenvalues of $\mathfrak{H} \Phi_{a}\left(t_{i}\right)$. This expression is supposed to reproduce the isolated-orbits approximation

$$
\Psi(E) \approx \sum_{\text {c.o. } i} \mathcal{A}_{i}(E) e^{i S_{i}(E)} .
$$

In this case, the sum extends over all closed orbits involved in the bifurcation that are real at the given energy $E$. If the normal form $\Phi_{a}(t)$ has been chosen suitably, there is a oneto-one correspondence between these orbits and the stationary points $t_{i}$. A comparison of Eqs. (44) and (45) yields the conditions

$$
S_{i}(E)=S_{0}(E)+\Phi_{a}\left(t_{i}\right)
$$

and

$$
\mathcal{A}_{i}(E)=\frac{(2 \pi i)^{n / 2} p\left(t_{i}\right)}{\sqrt{\left|\mathfrak{H} \Phi_{a}\left(t_{i}\right)\right|}} e^{-i \pi v_{i} / 2} .
$$

These equations must be valid for real orbits. In most bifurcation scenarios, all orbits are real at least at certain energies. In these cases, it appears natural to postulate (47) also to hold for ghost orbits. The parameter values one obtains are then smooth functions of the energy even at the bifurcations where the orbits become ghosts. In some instances, bifurcations involving only ghost orbits occur $[39,40]$. In these cases, condition (47) still produces smoothly varying parameters and enforces the desired asymptotics.

The numbers $\nu_{i}$ of negative eigenvalues change discontinuously at a bifurcation. For orbits that are real on either side of the bifurcation, so do the Maslov indices contained in the semiclassical amplitudes $\mathcal{A}_{i}$. These changes must compensate each other if the values $p\left(t_{i}\right)$ are to be continuous across the bifurcation. For these orbits, therefore, the change of the Maslov index occurring in a bifurcation must be equal to the change in $\nu_{i}$ and can be determined from the normal form. For ghost orbits, the Maslov indices are not well defined classically. They must be chosen such as to make $p\left(t_{i}\right)$ continuous.

The normal form parameters $a$ and the action $S_{0}(E)$ can be determined from Eq. (46). They usually turn out to be unique. The amplitude function $p(t)$, on the contrary, is unknown. Once the parameters $a$ have been found, Eq. (47) specifies its values $p\left(t_{i}\right)$ at the stationary points of $\Phi_{a}(t)$. These values, of course, do not suffice to identify $p(t)$ uniquely, so that there is considerable freedom in the choice of $p(t)$. Usually, if there are $k$ orbits participating in the bifurcation scenario, we will approximate $p(t)$ by a polynomial of degree $k-1$. This choice is justified by the observation that the uniform approximation is needed only close to a bifurcation, where all orbits are close to $t=0$. Thus, in the spirit of the stationary-phase approximation, the dominant contributions to integral (43) stem from the neighborhood of $t=0$, whereas the regions of large $t$ do not contribute. A suitable approximation to $p(t)$ must therefore be precise close to the origin. This is achieved by a Taylor series expansion that leads to the polynomial ansatz.

Simple as it might appear, however, this choice can bring about a mathematical difficulty: A polynomial $p(t)$ diverges as $t \rightarrow \infty$, so that there is no guarantee that integral (43) will converge. If it does not, its divergence is an artifact of the choice of $p(t)$, because by construction the regions of large $t$ should not significantly influence the value of the integral. In this case, a suitable regularization scheme must be applied. It can be justified by verifying that the regularized integral possesses the correct asymptotics.

A slightly simpler form of the uniform approximation is obtained if the function $p(t)$ is assumed to be a constant. This approximation does not exactly reproduce the desired asymptotics, but as the transition across the bifurcation mainly results in a change of the stationary points of $\Phi_{a}(t)$ rather than essential changes in $p(t)$, it can be expected to capture the principal features.

It is clear from the above description that there is a certain arbitrariness in the procedure. This arbitrariness can be reduced to the choice of a suitable amplitude function $p(t)$, because by the splitting lemma and the classification theorems of catastrophe theory [41] the uniform approximation can always be brought into form (42) by a suitable coordinate transformation, provided a normal form $\Phi_{a}(t)$ equivalent to the actual action function is given.

In the following sections, uniform approximations for the two generic codimension-1 bifurcations described in Ref. [17] will be derived along the lines given here. They turn out to be analogous to those for isochronous and period-doubling bifurcations of periodic orbits given by Schomerus and Sieber [33].

\section{B. The fold catastrophe uniform approximation}

The simplest closed-orbit bifurcation is the creation of two orbits in a tangent bifurcation. It is described by the fold catastrophe

$$
\Phi_{a}(t)=\frac{1}{3} t^{3}-a t
$$

This normal form has stationary points at $t= \pm \sqrt{a}$, which are real if $a>0$. Its stationary values are

$$
\Phi( \pm \sqrt{a})=\mp \frac{2}{3} a^{3 / 2} .
$$

By Eq. (46), the actions $S_{1}$ and $S_{2}$ of the bifurcating orbits must satisfy

$$
\begin{aligned}
& S_{1}=S_{0}(E)-\frac{2}{3} a^{3 / 2}, \\
& S_{2}=S_{0}(E)+\frac{2}{3} a^{3 / 2} .
\end{aligned}
$$

For these equations to hold, one must assume $S_{1}<S_{2}$ if the orbits are real and $\operatorname{Im} S_{1}>0, \operatorname{Im} S_{2}<0$ if they are ghosts. These conditions determine how the orbits are to be associated with the stationary points of $\Phi_{a}(t)$. Equation (50) can be solved for 


$$
S_{0}(E)=\frac{S_{1}+S_{2}}{2}
$$

and

$$
|a|=\left(\frac{3}{4}\left|S_{2}-S_{1}\right|\right)^{2 / 3} \text {. }
$$

The observation that the bifurcating orbits are real if $a>0$ and ghosts if $a<0$ fixes the sign of $a$. Both $S_{0}(E)$ and $a$ have thus been determined.

For the semiclassical amplitudes, Eq. (47) yields

$$
\begin{aligned}
& \mathcal{A}_{1}=\frac{\sqrt{\pi}}{|a|^{1 / 4}} p(+\sqrt{a}) e^{+i \pi / 4}, \\
& \mathcal{A}_{2}=\frac{\sqrt{\pi}}{|a|^{1 / 4}} p(-\sqrt{a}) e^{-i \pi / 4} .
\end{aligned}
$$

With the ansatz

$$
p(t)=\frac{p_{0}}{2 \pi}+\frac{p_{1}}{2 \pi} t
$$

for the amplitude function $p(t)$, we can solve for the parameters $p_{0}$ and $p_{1}$ to obtain

$$
\begin{aligned}
& p_{0}=\sqrt{\pi}|a|^{1 / 4} e^{-i \pi / 4}\left(\mathcal{A}_{1}+i \mathcal{A}_{2}\right), \\
& p_{1}=\sqrt{\pi} \frac{|a|^{1 / 4}}{\sqrt{a}} e^{-i \pi / 4}\left(\mathcal{A}_{1}-i \mathcal{A}_{2}\right) .
\end{aligned}
$$

The uniform approximation thus takes the form

$$
\Psi(E)=\left(p_{0} I_{0}+p_{1} I_{1}\right) e^{i S_{0}(E)}
$$

with

$$
I_{k}=\frac{1}{2 \pi} \int d t t^{k} e^{i \Phi_{a}(t)}
$$

The integral $I_{0}$ can be evaluated in terms of the Airy function [24] as

$$
I_{0}=\operatorname{Ai}(-a),
$$

whereas $I_{1}$ is given by its derivative

$$
I_{1}=i \frac{d}{d a} I_{0}=-i \mathrm{Ai}^{\prime}(-a)
$$

With these results, uniform approximation (56) can be computed once the classical quantities $S_{1}, S_{2}$ and $\mathcal{A}_{1}, \mathcal{A}_{2}$ are known. After some rearrangements, Eq. (56) can be found to agree with the uniform approximation derived by Schomerus and Sieber [33] for isochronous bifurcations of periodic orbits, although its present form is much simpler.

\section{The cusp catastrophe uniform approximation}

The normal form for the symmetrized cusp catastrophe is given by

$$
\Phi_{a}(t)=\frac{1}{4} t^{4}-\frac{1}{2} a t^{2}
$$

It has stationary points at $t=0$ and $t= \pm \sqrt{a}$ and describes a pitchfork bifurcation, where two asymmetric orbits bifurcate off an orbit invariant under a reflection. We denote their actions and amplitudes by $S_{\text {sym }}, S_{\text {asym }}$ and $\mathcal{A}_{\text {sym }}, \mathcal{A}_{\text {asym }}$, respectively, where $\mathcal{A}_{\text {asym }}$ is understood to be the cumulative amplitude of both asymmetric orbits.

As $\Phi_{a}(t=0)=0$, the reference action $S_{0}(E)$ must be chosen equal to the action of the symmetric orbit. The action difference is given by the stationary value of $\Phi_{a}(t)$, which is $a^{2} / 4$, so that

$$
\Delta S=S_{\text {sym }}-S_{\text {asym }}=\frac{1}{4} a^{2},
$$

and

$$
a= \pm 2 \sqrt{\Delta S} \text {. }
$$

The parameter $a$ has to be chosen positive if the asymmetric orbits are real, and negative otherwise. Here, $\Delta S$ was assumed to be positive. If it is not, the normal form $\Phi_{a}(t)$ must be replaced with $-\Phi_{a}(t)$, which changes the sign of the stationary values.

Due to the reflection symmetry, the amplitude function must be an even function of $t$. We make the ansatz

$$
p(t)=p_{0}+p_{2} t^{2}
$$

and solve Eq. (47) for the coefficients

$$
\begin{gathered}
p_{0}=\sqrt{\frac{a}{2 \pi}} \mathcal{A}_{\mathrm{sym}} e^{i \pi / 4}, \\
p_{2}=\frac{e^{-i \pi / 4}}{2 \sqrt{\pi a}}\left(\mathcal{A}_{\mathrm{asym}}-\sqrt{2} i \mathcal{A}_{\mathrm{sym}}\right) .
\end{gathered}
$$

The complete uniform approximation reads

$$
\Psi(E)=\int d t p(t) e^{i \Phi_{a}(t)}=p_{0} I_{0}+p_{2} I_{2}
$$

with

$$
I_{k}=\int d t t^{k} e^{i \Phi_{a}(t)}
$$

The integral $I_{0}$ can be evaluated analytically in terms of the Bessel functions [42]:

$$
I_{0}=\frac{\pi}{2} \sqrt{|a|} e^{-i a^{2} / 8}\left[e^{i \pi / 8} J_{-1 / 4}\left(\frac{a^{2}}{8}\right)+\operatorname{sgn} a e^{-i \pi / 8} J_{1 / 4}\left(\frac{a^{2}}{8}\right)\right] \text {. }
$$


Although it is not apparent at first sight, $I_{0}$ is a smooth function of $a$. This can be verified if the series expansion [24]

$$
J_{\nu}(x)=\left(\frac{x}{2}\right)^{\nu} r_{\nu}(x)
$$

with $r_{\nu}(x)$ a power series in $x^{2}$ is used. In terms of $r_{\nu}(x)$,

$$
I_{0}=\frac{\pi}{2} e^{-i a^{2} / 8}\left[2 e^{i \pi / 8} r_{-1 / 4}\left(\frac{a^{2}}{8}\right)+\frac{a}{2} e^{-i \pi / 8} r_{1 / 4}\left(\frac{a^{2}}{8}\right)\right],
$$

which is indeed smooth. The second integral $I_{2}$ can be evaluated from

$$
\begin{aligned}
I_{2}= & \int d t 2 i \frac{d}{d a} e^{i \Phi_{a}(t)}=2 i \frac{d I_{0}}{d a}=i \pi \sqrt{|a|} e^{-i a^{2} / 8}\left\{\left(\frac{1}{2 a}-i \frac{a}{4}\right)\right. \\
& \times\left[e^{i \pi / 8} J_{-1 / 4}\left(\frac{a^{2}}{8}\right)+\operatorname{sgn} a e^{-i \pi / 8} J_{1 / 4}\left(\frac{a^{2}}{8}\right)\right] \\
& +\frac{a}{8} e^{i \pi / 8}\left[J_{-5 / 4}\left(\frac{a^{2}}{8}\right)-J_{3 / 4}\left(\frac{a^{2}}{8}\right)\right] \\
& \left.+\operatorname{sgn} a \frac{a}{8} e^{-i \pi / 8}\left[J_{-3 / 4}\left(\frac{a^{2}}{8}\right)-J_{5 / 4}\left(\frac{a^{2}}{8}\right)\right]\right\} .
\end{aligned}
$$

This derivation contains an interchange of differentiation and integration, which achieves a regularization of the divergent integral $I_{2}$. It can be justified by verifying that the asymptotic behavior of Eq. (70) for $a \rightarrow \pm \infty$ agrees with the stationary-phase approximation to Eq. (66).

\section{UNIFORMIZED RECURRENCE SPECTRA}

The formulas derived in the preceding sections give the uniform approximations directly in terms of the semiclassical actions and amplitudes. This circumstance makes them easy to apply to scaled spectra: we simply put $S=w \widetilde{S}$ and $\mathcal{A}_{\text {c.o. }}$. $=w^{-1} \widetilde{\mathcal{A}}_{\text {c.o. }}$. As $w$ is varied, the bifurcation is not encountered because the classical mechanics does not change, so that the isolated-orbits approximation does not actually diverge. However, if $w$ is small, the action differences between the bifurcating orbits are also small, so that the presence of the bifurcation is felt and the isolated-orbits formula produces exceedingly large contributions. For large $w$, the action differences also grow large, so that the isolated-orbits approximation should be recovered in the limit of large $w$.

These findings are illustrated in Fig. 7 for a pitchfork bifurcation taking place in the first series of rotators at a repetition number $\mu=57$. At $\widetilde{E}=-1.4$, the bifurcation takes place at the scaled electric field strength $\widetilde{F}=0.09014$. The data shown in Fig. 7 were calculated for $\widetilde{E}=-1.4$ and $\widetilde{F}$ $=0.2$, which are sufficiently far away from the bifurcation for the asymptotic regime to be reached within the range of $w$ shown. As anticipated, in the limit of $w \rightarrow \infty$ the complete uniform approximation agrees with the isolated-orbits formula. The simple approximation also reproduces the beats correctly, but it has a smaller amplitude.

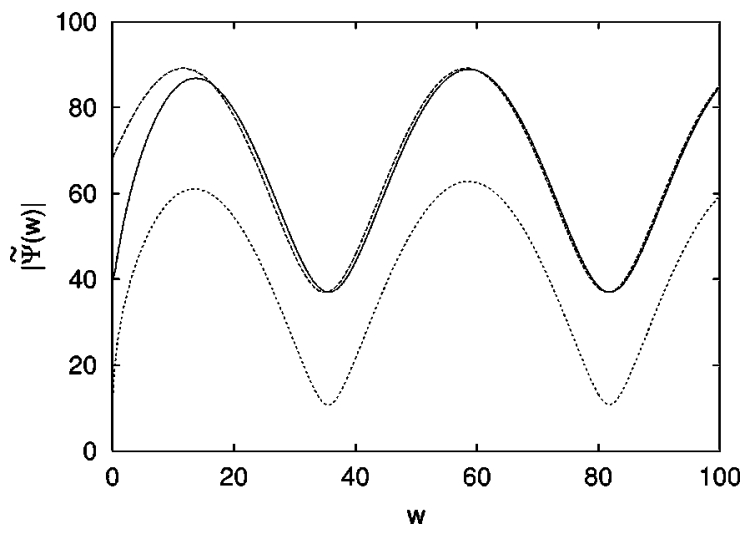

FIG. 7. Uniform approximation for a scaled spectrum (see text for a description of the bifurcation). Solid line: uniform approximation [Eq. (65)]. Short-dashed line: simple uniform approximation with constant $p(t)$. Long-dashed line: isolated-orbits approximation.

The scaled uniform approximation can be used to improve the semiclassical recurrence spectrum, but this requires some effort. Although the isolated-orbits approximation yields $\delta$ function peaks in the recurrence spectrum, which are replaced with Gaussians due to the smoothing of the recurrence spectrum (see Sec. VII), the uniform approximation is a complicated function of $w$. It must be subjected to a numerical Fourier transform in the same way as the quantum spectrum if its contribution to the recurrence spectrum is to be evaluated. Because a bifurcation involves orbits with roughly equal actions, the uniform approximation will produce a recurrence peak at the appropriate action. An example is shown in Fig. 8. It was calculated for the bifurcation already described in Fig. 7. The Gaussian smoothing used in Sec. VII was replaced with a rectangular window, so that a number of side peaks appear. In this case, the Fourier transform of both the uniform approximation and the isolatedorbits approximation was taken over the rectangular window $w \in[40,60]$. The bifurcating orbits have the scaled action $\widetilde{S} / 2 \pi \approx 21.86$, which is where the Fourier peaks are centered

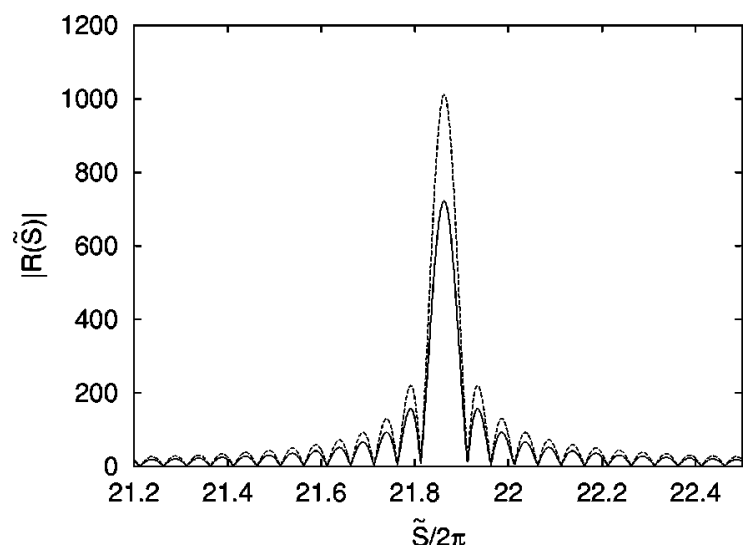

FIG. 8. Contribution to the recurrence peak calculated from the uniform approximation (solid line) and the isolated-orbits approximation (dashed line) for the same bifurcation as in Fig. 7, $\widetilde{E}$ $=-1.4$ and $\widetilde{F}=0.1$. 
in both approximations. The peak produced by the uniform approximation is considerably smaller.

If this uniformization procedure is carried out for all excessively high bifurcation peaks, it should be possible to bring the semiclassical recurrence spectrum in Fig. 6 into agreement with its quantum counterpart. In practice, however, several obstacles stand in the way. First of all, in many cases ghost orbits must be included in the uniform approximation. They must be found and identified as pertinent to a given bifurcation before the uniformization can be performed. Furthermore, even if all relevant orbits are real, those orbits connected with each other in a bifurcation must be recognized in the data set. This is by no means an easy task. For example, if in a given series of rotators and for a given winding number a quartet of orbits appears, there are two different doublet orbits out of which they may have bifurcated, and it is not clear, in general, which of them must be taken for the uniform approximation. In a single case, this can be found out fairly comfortably by hand. If many orbits are to be classified, however, it is essential to do the grouping automatically. We have not yet been able to devise a practical algorithm for this task, so that an automatized uniformization of all bifurcation peaks is presently impossible.

Apart from these rather technical difficulties, there are also some obstacles of more fundamental importance. Consider, e.g., the two high semiclassical peaks at $\widetilde{S} / 2 \pi \approx 25$ in Fig. 5. They are notably too high, and they are well isolated from neighboring recurrence peaks, so that they may appear to be the ideal testing ground for the uniformization procedure. These peaks are generated by vibrators with repetition numbers $\mu=41$ and $\mu=42$, respectively. The pertinent bifurcation scenarios were described in Figs. 17 and 18 of Ref. [17]. The "simple" scenario taking place at $\mu=41$ consists of two orbits being generated in the rotational symmetry breaking at $\widetilde{F}=0$, followed by a tangent bifurcation destroying one of them and a third orbit. To smooth this bifurcation peak, a uniform approximation describing the complete scenario must be found, which requires the construction of a suitable normal form. Although a uniform approximation for the symmetry-breaking is available [43-45], the derivation of the pertinent normal form relies on principles different from the catastrophe theory classification used here, and it is not clear how these two can be united into a single normal form. Thus, the construction of a uniform approximation for this bifurcation scenario, and even more so for the more complicated scenario at $\mu=42$, remains an open problem to be solved in the future. It can be solved within the framework of uniformization presented in Sec. VIII A, but will require a way of constructing normal forms.

The approach to high-resolution semiclassical quantization relies on the harmonic inversion of a Fourier transformed semiclassical spectrum, i.e., of a recurrence spectrum. The above method of uniformizing the bifurcationinduced excessively high recurrence peaks in a semiclassical spectrum would therefore, if it could be implemented systematically, also pave the way for the inclusion of uniform approximations into a high-resolution semiclassical quantization, which has not been possible so far. We were able to demonstrate the feasibility of our method by way of example for the hydrogen atom in an electric field [46], which is less demanding classically. Its application to the crossed-fields hydrogen atom, however, remains open for future work.

\section{CONCLUSION}

For the first time, a high-resolution semiclassical quantization of the hydrogen atom in crossed electric and magnetic fields has been presented. It achieved the identification of the strong spectral lines in different $n$ manifolds. By means of a detailed semiclassical analysis of the pertinent quantum spectrum, it was shown that bifurcations of closed orbits play a crucial role in the semiclassical spectrum and preclude the resolution of finer details in the semiclassical spectrum. They pose a particular challenge to the semiclassical quantization because they require a special treatment by uniform approximations.

A simple heuristic scheme for the construction of uniform approximations has been proposed. Its simplicity and efficacy was demonstrated by a derivation of the uniform approximations for the codimension- 1 generic bifurcations of closed orbits.

We have devised a general method for the inclusion of uniform approximations in a high-resolution semiclassical quantization by harmonic inversion. In a recent publication [46] it was successfully applied to the hydrogen atom in an electric field. In the case of the crossed-fields hydrogen atom, the diversity and complexity of the bifurcation scenarios encountered so far hinders the systematic implementation of the uniformization procedure. The treatment of all relevant bifurcations and the calculation of a detailed semiclassical spectrum thus remain challenging tasks for future studies.
[1] M.L. Du and J.B. Delos, Phys. Rev. A 38, 1896 (1988).

[2] E.B. Bogomolny, Zh. Eksp. Teor. Fiz. 96, 487 (1989) [Sov. Phys. JETP 69, 275 (1989)].

[3] J. Gao, J.B. Delos, and M. Baruch, Phys. Rev. A 46, 1449 (1992).

[4] J.-M. Mao, K.A. Rapelje, S.J. Blodgett-Ford, and J.B. Delos, Phys. Rev. A 48, 2117 (1993).

[5] M. Courtney, Phys. Rev. A 51, 4558 (1995).

[6] J. Main, Ph. D. thesis, Universität Bielefeld, Germany, 1991.

[7] G. Raithel, M. Fauth, and H. Walther, Phys. Rev. A 44, 1898
(1991).

[8] A.D. Peters and J.B. Delos, Phys. Rev. A 47, 3020 (1993).

[9] B. Hüpper, J. Main, and G. Wunner, Phys. Rev. A 53, 744 (1996).

[10] K. Weibert, J. Main, and G. Wunner, Ann. Phys. (N.Y.) 268, 172 (1998).

[11] P.A. Dando, T.S. Monteiro, D. Delande, and K.T. Taylor, Phys. Rev. Lett. 74, 1099 (1995).

[12] P.A. Dando, T.S. Monteiro, D. Delande, and K.T. Taylor, Phys. Rev. A 54, 127 (1996). 
[13] A. Matzkin and T.S. Monteiro, Phys. Rev. Lett. 87, 143002 (2001).

[14] J. Main, V.A. Mandelshtam, G. Wunner, and H.S. Taylor, Nonlinearity 11, 1015 (1998).

[15] J. Main, Phys. Rep. 316, 233 (1999).

[16] J. Main and G. Wunner, Phys. Rev. A 59, R2548 (1999).

[17] T. Bartsch, J. Main, and G. Wunner, Phys. Rev. A 67, 063410 (2003) (preceding paper).

[18] B.E. Granger and C.H. Greene, Phys. Rev. A 62, 012511 (2000).

[19] M. Aymar, C.H. Greene, and E. Luc-Koenig, Rev. Mod. Phys. 68, 1015 (1996).

[20] F. Robicheaux, Phys. Rev. A 48, 4162 (1993).

[21] T. Bartsch, The Hydrogen Atom in an Electric Field and in Crossed Electric and Magnetic Fields: Closed-Orbit Theory and Semiclassical Quantization (Cuvillier, Göttingen, Germany, 2002).

[22] M. C. Gutzwiller, Chaos in Classical and Quantum Mechanics (Springer-Verlag, New York, 1990).

[23] T. Bartsch, e-print physics/0301017.

[24] M. Abramowitz and I. A. Stegun, Pocketbook of Mathematical Functions (Verlag Harri Deutsch, Frankfurt/Main, 1984).

[25] J. Rao, D. Delande, and K.T. Taylor, J. Phys. B 34, L391 (2001).

[26] T. Bartsch, J. Main, and G. Wunner, Phys. Rev. E 64, 056705 (2001).

[27] J. Main, P.A. Dando, Dž. Belkić, and H.S. Taylor, J. Phys. A 33, 1247 (2000).

[28] J. Main, K. Weibert, V.A. Mandelshtam, and G. Wunner, Phys. Rev. E 60, 1639 (1999).

[29] J.N.L. Connor, Mol. Phys. 26, 1217 (1973).

[30] J.N.L. Connor, Mol. Phys. 31, 33 (1976).
[31] A.M. Ozorio de Almeida and J.H. Hannay, J. Phys. A 20, 5873 (1987).

[32] M. Sieber, J. Phys. A 29, 4715 (1996).

[33] H. Schomerus and M. Sieber, J. Phys. A 30, 4537 (1997).

[34] M. Sieber and H. Schomerus, J. Phys. A 31, 165 (1998).

[35] J. Main and G. Wunner, Phys. Rev. A 55, 1743 (1997).

[36] M. Kuś, F. Haake, and D. Delande, Phys. Rev. Lett. 71, 2167 (1993).

[37] H. Schomerus, Europhys. Lett. 38, 423 (1997).

[38] H. Schomerus, J. Phys. A 31, 4167 (1998).

[39] T. Bartsch, J. Main, and G. Wunner, J. Phys. A 32, 3013 (1999).

[40] T. Bartsch, J. Main, and G. Wunner, Ann. Phys. (N.Y.) 277, 19 (1999).

[41] D. P. L. Castrigiano and S. A. Hayes, Catastrophe Theory (Addison-Wesley, Reading, 1993).

[42] I. S. Gradshteyn and I. M. Ryzhik, Table of Integrals, Series, and Products (Academic, New York, 1965).

[43] S. Tomsovic, M. Grinberg, and D. Ullmo, Phys. Rev. Lett. 75, 4346 (1995).

[44] D. Ullmo, M. Grinberg, and S. Tomsovic, Phys. Rev. E 54, 136 (1996).

[45] M. Sieber, J. Phys. A 30, 4563 (1997).

[46] T. Bartsch, J. Main, and G. Wunner, Phys. Rev. A 66, 033404 (2002).

[47] The regular and irregular Coulomb functions $f$ and $g$ used by Granger and Greene [18] differ from those used by Robicheaux [20] in that they are energy normalized in Rydberg rather than in Hartree units. The radial function matrices given here agree in normalization with those adopted by Robicheaux, whereas the matrices used by Granger and Greene are inconsistent with their Eq. (12). 